\title{
Diabetes mellitus y obesidad: la importancia de realizar un abordaje integral de ambas entidades
}

\begin{abstract}
Miguel Martín-Romero, Miriam Navarro-Rodríguez, José David Galián-Ramírez, Raquel Pérez-Luján, Antonia María Castillo-Navarro Hospital Clínico Universitario Virgen de la Arrixaca. Murcia. España
\end{abstract}

Recibido: 6/11/2018

Aceptado: 01/01/2019

En línea: 31/01/2019

Citar como: Martín-Romero M, Navarro-Rodríguez M, Galián-Ramírez JD, Pérez-Luján R, Castillo-Navarro AM. Diabetes mellitus y obesidad: la importancia de realizar un abordaje integral de ambas entidades. Rev Esp Casos Clin Med Intern (RECCMI). 2019 (Ene); 4(Supl 1): 5-6. doi: 10.32818/reccmi.a4s1a3.

Autor para correspondencia: Miguel Martín-Romero. miguelmr1990@gmail.com

\section{Palabras clave \\ $\triangleright$ Diabetes mellitus tipo 2 \\ $\triangleright$ Obesidad \\ $\triangleright$ Agonistas del receptor GLP-1 \\ $\triangleright$ Inhibidores SGLT2}

\begin{abstract}
Resumen
Se presenta el caso de una mujer de 63 años diagnosticada de diabetes mellitus tipo 2 y obesidad grado III, muy limitada para la realización de ejercicio físico. Durante años, no se consiguió un control glucémico óptimo con antidiabéticos orales ni una reducción del peso a pesar de seguir varias dietas pautadas por especialistas. Además, rechazó sistemáticamente iniciar tratamiento insulínico. Ambos objetivos se lograron finalmente con un tratamiento combinado formado por agonistas del receptor GLP-1 e inhibidores SGLT2, todo ello con buena tolerancia y sin aparición de efectos adversos reseñables.

Abstract
We present a 63-year-old woman diagnosed with type 2 diabetes mellitus in 2007 and grade III obesity, in addition
to gonarthrosis and sequelae of a traffic accident. She was unable to perform physical exercise due to gonarthrosis
and the aftermath of the traffic accident. Progressive treatment with oral antidiabetic agents did not achieve opti-
mal glycemic control after several years of follow-up. Neither there was an improvement in obesity despite following
several diets prescribed by specialists. We offered her therapy with insulin but the patient rejected it systematically.
Finally, the use of combined treatment with agonists of the GLP-1 receptor and SGLT-2 inhibitors allowed to achieve
an adequate glycemic control for the first time. In the subsequent controls, HbAlc values of less than 7\% were reached,
in addition to a significant reduction in body weight, with good tolerance and without adverse effects.
\end{abstract}

Puntos destacados

$\triangleright$ En el manejo de la diabetes mellitus tipo 2 es fundamental emplear todas las herramientas a nuestro alcance para controlar el peso corporal de los pacientes.

$\triangleright$ Existen hoy en día fármacos con efecto significativo demostrado tanto a nivel de control glucémico como de reducción de peso.

\section{Introducción}

La diabetes mellitus (DM) tipo 2 constituye una de las enfermedades con mayor incidencia y prevalencia en la actualidad y está íntimamente ligada a la obesidad. Hoy en día, alrededor del 50\% de los pacientes diabéticos son también obesos, proporción que se espera que siga aumentando en los próximos años. Pese a que las actuaciones sobre el estilo de vida (dieta y ejercicio) constituyen la primera línea de tratamiento, resultan con frecuencia insuficientes y es necesario añadir tratamiento farmacológico para conseguir un adecuado control glucémico. En el momento actual, disponemos de un arsenal terapéutico variado capaz de actuar de forma simultánea a distintos niveles, permitiendo así tanto un mejor control de la enfermedad como reducciones significativas de peso corporal.

\section{Historia clínica: antecedentes, enfermedad actual y exploración física}

Mujer de 63 años, sin alergias medicamentosas conocidas, que presentaba los siguientes factores de riesgo cardiovascular: hipertensión arterial y cardiopatía hipertensiva en tratamiento con cuatro fármacos, dislipemia en tratamiento con estatinas, DM tipo 2 diagnosticada en 2007 en tratamiento con dieta y antidiabéticos orales, y obesidad grado III. Además, era fumadora hasta hacía 5 años de un paquete de tabaco al día desde la juventud. Como otros antecedentes personales de interés, estaba diagnosticada de enfermedad pulmonar obstructiva crónica (EPOC) en tratamiento inhalador con doble broncodilatación, presentaba gonartrosis bilateral y estaba intervenida de fractura de tibia derecha como consecuencia de accidente de tráfico hacía unos 10 años. Dichos factores, junto con la importante obesidad de la paciente, limitaban 
de forma importante su movilidad, imposibilitando la realización de ejercicio físico adecuado.

En el momento del diagnóstico de la diabetes, presentaba una hemoglobina glicosilada (HbA1c) de 9,2\% y un peso corporal de $112 \mathrm{~kg}$, lo que asociado a su estatura (1,55 m) constituía una obesidad grado III o mórbida (índice de masa corporal [IMC] 47). Además, la paciente se encontraba muy limitada para la realización de actividad física, uno de los pilares fundamentales de las medidas higiénico-dietéticas establecidas para el control de la enfermedad. Su médico de Atención Primaria decidió iniciar tratamiento farmacológico con metformina 850 mg/12 h, que debió suspender al poco tiempo por intolerancia gastrointestinal. Tras ello, decidió remitir a la paciente a Medicina Interna (Unidad de Riesgo Cardiovascular) para optimizar el tratamiento.

\section{Pruebas complementarias}

Desde la Unidad de Riesgo Cardiovascular se decidió entonces iniciar tratamiento combinado con glimepirida 2 mg/día y pioglitazona 30 mg/día, siendo seguida periódicamente en consultas externas durante los años siguientes. En 2008, su HbA1c era de 8,1\%; y en 2010, de 8,3\%, encontrándose en todo momento lejos de alcanzar el objetivo control ( $\mathrm{HbA} 1 \mathrm{c}<7 \%)$. Su peso osciló durante ese tiempo entre 110-120 kg, a pesar de comenzar varias dietas prescritas por especialistas. La actividad física continuaba siendo casi nula. En 2010, se añadió al tratamiento linagliptina 5 mg/día, pero en 2012 aún mantenía cifras de $\mathrm{HbA} 1 \mathrm{c}$ de 8,2\% y, por primera vez, se le sugirió la necesidad de iniciar insulinización, a lo que se negó sistemáticamente por miedo a un mayor aumento de peso. Al inicio del año 2015, su peso era de 116 kg (siguió oscilando alrededor de dichas cifras) y la HbA1c de 8,3\%. Se insistió entonces en la necesidad de iniciar tratamiento con insulina para mejorar el control glucémico, pero la paciente se negó nuevamente.

\section{Evolución}

Se planteó entonces modificar el tratamiento prescrito, suspendiendo linagliptina e iniciando administración de liraglutida subcutánea diaria, un agonista del receptor glucagon-like peptide-1 (GLP-1). La tolerancia al nuevo fármaco resultó adecuada en todo momento. Fue revisada en consultas externas al cabo de 6 meses, observándose una importante mejoría en el control de la diabetes y en el peso corporal. La HbA1c descendió de 8,3\% a 7,3\% y el peso se redujo de 114 kg a 106 kg. Para conseguir el objetivo de control glucémico establecido $(\mathrm{HbA} 1 \mathrm{c}<7 \%)$ se decidió en dicha visita añadir canagliflozina 100 mg/día un inhibidor del cotransportador sodio-glucosa 2 (iSGLT2). Tras el inicio de dicho fármaco, la paciente presentó buena tolerancia al tratamiento, sin signos de depleción de volumen o infecciones de tracto urinario, los efectos adversos más frecuentemente descritos en los iSGLT2.

En noviembre de 2015, a los 6 meses del inicio de canagliflozina, la paciente acudió a consultas externas de la Unidad de Riesgo Cardiovascular presentando por primera vez desde su diagnóstico una $\mathrm{HbA} 1 \mathrm{c}$ inferior a 7\% (6,9\%, lo que supone una reducción de 0,4\% con respecto a la visita de junio de 2015) y un peso de $100 \mathrm{~kg}$ (disminución de otros 6 kg). La tolerancia a ambos fármacos fue buena y no se produjo ningún efecto adverso reseñable. Por primera vez se consiguió un adecuado control de la enfermedad, ligado muy directamente a la significativa pérdida de peso.

\section{Diagnóstico}

- DM tipo 2

- Obesidad grado III.

\section{Discusión y conclusiones}

Actualmente, más del $80 \%$ de los pacientes con DM tipo 2 padecen sobrepeso u obesidad. El control del peso corporal constituye un pilar fundamental en el manejo de la enfermedad', aunque es bien conocida la dificultad para lograrlo. En numerosas ocasiones esto se complica por la dificultad para realizar una actividad física adecuada dada la patología de base de muchos pacientes, así como por la falta de motivación para llevar a cabo la dieta y ejercicio físico necesarios.

La aparición en los últimos años de tratamientos antidiabéticos con efectos significativos sobre el control del peso corporal supone una atractiva y eficaz opción terapéutica para este perfil de individuos². En el caso clínico presentado, se objetiva de manera clara el efecto sinérgico de dos de estos fármacos (un agonista del receptor GLP-1 y un iSGLT2) en el control del peso y de los niveles glucémicos medidos a través de la $\mathrm{HbA} 1 \mathrm{c}$, potenciándose ambos entre sí. En el momento actual, se dispone de un amplio abanico terapéutico que permite optimizar el tratamiento de la diabetes sin asociar ganancia de peso, aspecto clave del manejo en los pacientes diabéticos obesos ${ }^{4}$.

\section{Bibliografía}

1. American Diabetes Association. Standards of medical care in diabetes-2014. Diabetes Care. 2014 Jan; 37 Suppl 1: S14-80. doi: 10.2337/dc14-S014.

2. Inzucchi SE, Bergenstal RM, Buse JB, Diamant M, Ferrannini E, Nauck M, et al. American Diabetes Association (ADA); European Association for the Study of Diabetes (EASD). Management of hyperglycemia in type 2 diabetes: a patientcentered approach: position statement of the American Diabetes Association (ADA) and the European Association for the Study of Diabetes (EASD). Diabetes Care. 2012 Jun; 35(6): 1364-1379. doi: 10.2337/dc12-0413. Epub 2012 Apr 19.

3. Abdul-Ghani MA, DeFronzo RA. Inhibition of renal glucose reabsorption: a novel strategy for achieving glucose control in type 2 diabetes mellitus. Endocr Pract. 2008 Sep; 14(6): 782-790.

4. Bennett WL, Maruthur NM, Singh S, Segal JB, Wilson LM, Chatterjee R, et al. Comparative effectiveness and safety of medications for type 2 diabetes: an update including new drugs and 2-drug combinations. Ann Intern Med. 2011 May 3; 154(9): 602-613. doi: 10.7326/0003-4819-154-9-20110503000336. Epub 2011 Mar 14. 